\title{
Recombinant Enolase of Trypanosoma cruzi as a Novel Vaccine Candidate against Chagas Disease in a Mouse Model of Acute Infection
}

\author{
Minerva Arce-Fonseca, ${ }^{1}$ María Cristina González-Vázquez, ${ }^{2}$ Olivia Rodríguez-Morales, ${ }^{1}$ \\ Verónica Graullera-Rivera, ${ }^{3}$ Alberto Aranda-Fraustro, ${ }^{3}$ Pedro A. Reyes, ${ }^{1}$ \\ Alejandro Carabarin-Lima $\mathbb{D}^{1},{ }^{1,4}$ and José Luis Rosales-Encina ${ }^{4}$

\begin{abstract}
${ }^{1}$ Departamento de Biología Molecular, Juan Badiano No. 1, Col. Sección XVI, Delegación Tlalpan, Instituto Nacional de Cardiología "Ignacio Chávez", 14080 Mexico City, Mexico

${ }^{2}$ Departamento de Biología Celular, Avenida Instituto Politecnico Nacional No. 2508, Col. San Pedro Zacatenco, Centro de Investigación y de Estudios Avanzados del Instituto Politecnico Nacional, 07360 Mexico City, Mexico

${ }^{3}$ Departamento de Anatomía Patológica, Juan Badiano No. 1, Col. Sección XVI, Delegación Tlalpan, Instituto Nacional de Cardiología "Ignacio Chávez", 14080 Mexico City, Mexico

${ }^{4}$ Departamento de Infectómica y Patogénesis Molecular, Avenida Instituto Politecnico Nacional No. 2508, Col. San Pedro Zacatenco, Centro de Investigación y de Estudios Avanzados del Instituto Politecnico Nacional, 07360 Mexico City, Mexico
\end{abstract}

Correspondence should be addressed to Alejandro Carabarin-Lima; ecoli_75@hotmail.com

Received 7 February 2018; Accepted 3 April 2018; Published 7 May 2018

Academic Editor: Giuseppe A. Sautto

Copyright (c) 2018 Minerva Arce-Fonseca et al. This is an open access article distributed under the Creative Commons Attribution License, which permits unrestricted use, distribution, and reproduction in any medium, provided the original work is properly cited.

\begin{abstract}
Trypanosoma cruzi is the protozoan parasite that causes Chagas disease, which is considered by the World Health Organization to be a neglected tropical disease. Two drugs exist for the treatment of Chagas disease, nifurtimox and benznidazole; they are only effective in the acute phase, and a vaccine is currently not available. In this study, we used the recombinant enolase from $T$. cruzi H8 strain (MHOM/MX/1992/H8 Yucatán) (rTcENO) and its encoding DNA (pBKTcENO) to immunize mice and evaluate their protective effects in an experimental murine model of acute phase infection. Our results showed that mice vaccinated with rTcENO or its encoding DNA were able to generate typical specific antibodies (IgG1, IgG2a, and IgG2b), suggesting that a mixed Th1/Th2 immune response was induced. The parasite burden in the blood was reduced to $69.8 \%$ and $71 \%$ in mice vaccinated with rTcENO and pBKTcENO, respectively. The group vaccinated with rTcENO achieved $75 \%$ survival, in contrast to the group vaccinated with pBKTcENO that showed no survival in comparison to the control groups. Moreover, rTcENO immunization elevated the production of IFN- $\gamma$ and IL-2 after the parasite challenge, suggesting that the Th1-type immune response was polarized. These results indicated that $\mathrm{rTcENO}$ could be used as a vaccine against Chagas disease.
\end{abstract}

\section{Introduction}

The intracellular protozoan parasite Trypanosoma cruzi is the etiologic agent of Chagas disease, considered as a neglected tropical disease [1]. Currently, approximately 5.7 million people are infected worldwide, more than 70.2 million people are at risk of contracting the disease [2], and 50,000 patients die each year as a result of the disease [3].
Because of its natural life cycle, involving mitotic division in reduviid insects, which then transmit the infection by feeding on the blood of different vertebrates, T. cruzi is considered to be a severe health problem in rural areas of Mexico and Central and South America, where these insects are endemic. Chagas disease is also a health problem in nonendemic countries, such as the United States of America (USA), Canada, Australia, Japan, France, Spain, 
and Switzerland [4-8]. In Mexico, as well as in other endemic countries, cases are becoming more common in urban areas. This shift in the epidemiology of Chagas disease is connected to the migration of people infected with T. cruzi, blood transfusion, vertical transmission (mother to child), and organ transplantation [9].

Chagas disease has two different phases: acute and chronic. The acute phase is usually subclinical. Most acute cases are asymptomatic, last for 6-12 weeks, and typically occur in childhood [10]. Two major forms of the disease are observed during the chronic phase: indeterminate or latent and symptomatic. A high percentage of chagasic patients remain in the indeterminate phase for 10 or 30 years or even for life. These patients usually have no clinical or physical signs of disease but display positive serology. Approximately $30-40 \%$ of infected individuals develop clinical symptoms, involving severe cardiomyopathy or gastrointestinal pathology, several years after the infection.

Nifurtimox and benznidazole are currently the only licensed drugs with proven efficacy specifically against Chagas disease. Both drugs have significant activity during the acute phase of the disease, causing parasitological cure in up to $80 \%$ of patients who were treated early $[11,12]$. In addition to chemotherapy, a vaccine may provide a suitable preventive measure to control the spread of the disease. Different parasite antigens have been tested for their effectiveness in controlling the infection. These immunizations, which were carried out at the level of DNA or recombinant proteins, showed different degrees of protection (e.g., reduced parasitemia and survival). In most immunization cases, a Thl-type immune response was observed. These genes and proteins were tested for vaccine trials in experimental models of infection, including cruzipain [13, 14], trans-sialidase [15, 16], amastigote surface protein-2 [17], LYT-1 [18], and paraflagellar rod protein [19] among others.

In recent years, several studies have shown that the "moonlighting" protein enolase is capable of generating a protective immune response against Plasmodium falciparum [20], Candida albicans [21], and Ascaris suum [22].

Enolase (2-phospho-D-glycerate hydrolase, EC 4.2.1.11) is a metalloenzyme that catalyzes the reversible dehydration of D-2-phosphoglycerate (PGA) to phosphoenolpyruvate (PEP) in both glycolysis and gluconeogenesis. Enolases, ranging from bacteria to higher vertebrates, show highly conserved amino acid sequences, particularly at the catalytic site. Consequently, enzymes from diverse species share similar kinetic properties. Enolase requires magnesium for both catalysis and dimer stabilization [23]. Furthermore, enolase that acts as a plasminogen receptor on the cell surface of certain pathogens $[24,25]$ has been implicated in nuclear functions, such as transcriptional regulation (as a repressor or activator) in protozoa [26-28], plants [29], and animal cells $[30,31]$. Enolase is also involved in the stress response [32], vacuolar fusion processes [33], and alternative molecular chaperone functions $[34,35]$.

Previously, we cloned and sequenced the gene encoding enolase from $T$. cruzi and performed immunological in silico assays. Our data showed that the resulting sequence had several predicted peptides for $\mathrm{B}$ cells and cytotoxic $\mathrm{T}$ lymphocytes (CTL), which suggested that enolase could be a good immunogen [36].

In the present study, we immunized mice with the recombinant protein $\mathrm{rTcENO}$ or the recombinant $\mathrm{pBKTcENO}$ DNA plasmid. We then challenged the immunized mice with a lethal dose of T. cruzi. The mice immunized with rTcENO showed typical immunoglobulins for Th1/Th2 immune responses, a significant reduction in the level of parasite burden in the blood, and $75 \%$ survival rate in comparison to the control groups. Moreover, the detection of IFN- $\gamma$, TNF (alpha and beta), and IL-2, but not IL-4, showed a polarized Th1 immune response when mice were challenged with $T$. cruzi. Conversely, mice immunized with pBKTcENO did not survive after challenge with a high inoculum of T. cruzi, despite a significant reduction in the level of parasitemia. We found that immunization with rTcENO, but not with pBKTcENO, induced substantial protection in mice, indicating that rTcENO could be a good candidate to develop a vaccine against Chagas disease.

\section{Materials and Methods}

2.1. Immunization and Trypanosoma cruzi Challenge. All mice (female $\mathrm{BALB} / \mathrm{c}$ mice $6-8$ weeks old) were randomly assigned into control or vaccinated groups of eight mice each in two independent experiments. The mice were immunized by intraperitoneal (i.p.) injection with $10 \mu \mathrm{g}$ of the recombinant protein (rTcENO) emulsified in Freund's complete adjuvant (CFA) (Sigma) and boosted twice with $10 \mu \mathrm{g}$ of the rTcENO in Freund's incomplete adjuvant (IFA) every 2 weeks. The control, nonvaccinated animals were mock immunized with PBS/adjuvant in the same schedule as the immunized mice (they received one injection with CFA and two with IFA). This i.p. route has been used in the mouse model for immunization of vaccines based on recombinant proteins, epitopes, or microvesicles, demonstrating its protective efficacy [37-41].

For DNA-based immunizations, $100 \mu \mathrm{g}$ of recombinant plasmid (pBKTcENO) or vector DNA (pBK-CMV) (Stratagene) was dissolved in $50 \mu \mathrm{L}$ of sterile PBS, injected intramuscularly (i.m.) in the tibialis anterioris muscle and boosted twice every 2 weeks [42].

Both vaccinated (rTcENO or pBKTcENO) and control mice (PBS or pBK-CMV) had access to food and water ad libitum, and two weeks after the last immunization, they received an i.p. injection of $8 \times 10^{4}$ bloodstream trypomastigotes of T. cruzi. The T. cruzi H8 strain (MHOM/MX/ 1992/H8 Yucatán (T. cruzi)) used in this work was a kind gift from Dr. Jorge E. Zavala Castro from the Centro de Investigaciones Regionales "Dr. Hideyo Noguchi”, Universidad Autónoma de Yucatán, Mérida, Yucatán, Mexico. Blood samples were collected from the tail vein to determine parasite burden every three days in the peripheral blood; in another similar experiment, survival rates were monitored daily. Mice were housed in a controlled environment and managed according to the National Institutes of Health Guide for Care and Use of Experimental Animals [43], with the approval of the CINVESTAV-IPN Animal Care and Use Committee. 
2.2. $r$ TcENO Polyclonal Antibodies. The recombinant protein $\mathrm{rTcENO}$ was obtained as previously described [36] and the purified rTcENO did not contain detectable levels of endotoxin contamination as measured by the E-Toxate assay (Sigma, St. Louis, MO, USA). Female BALB/c mice (6-8 weeks old) were immunized with $10 \mu \mathrm{g} /$ mouse. Each animal received three i.p. doses of antigen every seven days; the first immunization dose was administered in complete Freund's adjuvant, and the following immunizations were administered in incomplete Freund's adjuvant. Before and at the end of the immunization scheme, animals were bled to collect serum.

\subsection{Plasmid DNA Construction and Purification. The gene} encoding TcENO (GenBank access number: KC862322) was obtained from the pRSETB-TcENO plasmid [36] as a $1.1 \mathrm{~kb}$ Kpn I/Hind III (New England Biolabs) fragment. This fragment was subcloned into the prokaryotic/eukaryotic expression vector pBK-CMV (InvitrogenTM by Life Technologies) to generate the pBKTcENO plasmid. The correct subcloning of the TcENO gene was confirmed by restriction enzyme analysis and sequencing.

Plasmid DNA was purified by anion-exchange chromatography using a Qiagen Plasmid Maxi Kit. DNA used for immunizations was sterilized by ethanol precipitation and resuspended in lipopolysaccharide-free PBS (Gibco).

Enolase was identified in T. cruzi total protein extracts or purified rTcENO by western blot.

Briefly, T. cruzi epimastigotes were harvested from cultures and resuspended in lysis buffer $(10 \mathrm{mM}$ Tris- $\mathrm{HCl}$, pH 7.5; $5 \mathrm{mM}$ EDTA; $1 \%$ Nonidet P-40; $1 \mathrm{mM}$ phenylmethanesulfonyl fluoride; $10 \mathrm{mg} / \mathrm{mL}$ aprotinin; $50 \mathrm{U} / \mathrm{L}$ trasylol; and $10 \mathrm{mg} / \mathrm{mL}$ leupeptin) by repeated freezing and thawing cycles. Lysates were cleared by centrifugation $\left(30 \mathrm{~min}, 4^{\circ} \mathrm{C}\right.$ at $14,000 \times \mathrm{g}$ ), and the supernatants were collected and resolved by $12 \%$ sodium dodecyl-sulfate polyacrylamide gel electrophoresis (SDS-PAGE, $10 \mu \mathrm{g}$ and $20 \mu \mathrm{g}$ per lane) or purified rTcENO (10 $\mu$ g per lane). Proteins were electrotransferred onto nitrocellulose membranes at $70 \mathrm{~V}$ for $1 \mathrm{~h}$. The membranes were blocked with 5\% $(w / v)$ skim milk in phosphate-buffered saline (PBS, $\mathrm{pH} 7.4$ ) for $1 \mathrm{~h}$ at $37^{\circ} \mathrm{C}$, washed three times with PBS containing 0.05\% Tween 20 (PBS-T), $10 \mathrm{~min} /$ per time, and then incubated overnight at $4^{\circ} \mathrm{C}$ with anti-rTcENO polyclonal antibodies (1:500 dilution in $2 \%$ skim milk-PBS) or anti-pBKTcENO polyclonal antibodies ( $1: 500$ dilution in $2 \%$ skim milk-PBS). The negative control consisted of a pool of serum from different healthy mice diluted 1:500 in PBS with 2\% nonfat milk. After washing, the membranes were incubated with secondary antibody conjugated with alkaline phosphatase (Zymed Lab) for $1 \mathrm{~h}$ at $37^{\circ} \mathrm{C}$ at 1:5000 dilution in $2 \%$ skim milk-PBS. The blots were visualized with nitro-blue tetrazolium chloride (NBT) and 5-bromo-4-chloro-3'-indolyphosphate p-toluidine salt (BCIP) (Sigma).

2.4. Indirect Immunofluorescence Assays. T. cruzi epimastigotes were washed three times in PBS supplemented with $0.1 \%$ glucose (PBSG), $\mathrm{pH} 7.4$, and fixed in $2 \%$ paraformaldehyde in PBS $(v / v)$ for $2 \mathrm{~h}$ at $4^{\circ} \mathrm{C}$. After that, $1 \times 10^{6}$ parasites/
$\mathrm{mL}$ were placed on glass slides for $45 \mathrm{~min}$ at $37^{\circ} \mathrm{C}$. These preparations were divided into two sets; one remained nonpermeabilized, and the other was permeabilized with $0.2 \%$ Triton X-100 in PBS. Both preparations were incubated with antibodies against either TcENO or T. cruzi total protein extract from mice serum (diluted 1:100 in PBS-5\% BSA) for $1 \mathrm{~h}$ at room temperature using an FITC-labelled IgG (Pierce) as a secondary antibody diluted 1:3000 in PBS. After $1 \mathrm{~h}$ at room temperature, we rinsed the slides in PBS, stained the nuclear and kinetoplast DNA with DAPI, and mounted them with Vecta-Shield medium (Vector Laboratories). The results were observed with a Carl Zeiss LSM 700 confocal microscope.

2.5. Immunoglobulin Determination. Total IgG immunoglobulin and isotypes IgG1, IgG2a, IgG2b, IgG3, IgA, and IgM were evaluated by the ELISA method according to the manufacturer's instructions (Zymed Labs). Briefly, plates were coated with rTcENO $(2 \mu \mathrm{g} / \mathrm{mL})$ in carbonate buffer ( $\mathrm{pH}$ 9.6) and incubated overnight at $4^{\circ} \mathrm{C}$. Plates were washed with PBS- $0.1 \%$ Tween (PBST), incubated for $2 \mathrm{~h}$ at $37^{\circ} \mathrm{C}$ with blocking solution (PBS containing 5\% skim milk), washed with PBST and PBS, and then incubated with $50 \mu \mathrm{L}$ of either mouse anti-rTcENO or anti-pBKTcENO (1:500 dilutions). As a negative control, a pool of preimmune sera was used in all experiments. After washing, the peroxidase-labeled rabbit anti-mouse IgG antibodies were added at 1:1000 dilution in PBST and incubated for $1 \mathrm{~h}$ at room temperature. Plates were incubated with $100 \mu \mathrm{L}$ of ABTS substrate (2,2,-azinobis[3-ethylbenzthiazoline]-6-sulphonic acid) (Zymed Labs) for $10 \mathrm{~min}$ and read at $405 \mathrm{~nm}$ in an ELISA microplate reader (MultisKan MS).

2.6. Cytokine Determination by Flow Cytometry. The levels of serum Th1- (INF- $\gamma$, IL-2, and TNF) and Th2- (IL-4) type cytokines were analyzed in duplicate using a fluorescent bead immunoassay for quantitative detection by flow cytometry (Mouse Th1/Th2 Cytokine Kit, BD ${ }^{\mathrm{TM}}$ Cytometric Bead Array (CBA); BD Biosciences) according to the manufacturer's instructions. Briefly, serum samples $(50 \mu \mathrm{L})$ and phycoerythrin- (PE-) conjugated antibodies were incubated with capture bead reagent for $2 \mathrm{~h}$ in the dark at room temperature. Unbound antibodies were washed $(1.0 \mathrm{~mL}$ wash buffer) and resuspended in $300 \mathrm{~mL}$ of PBS. Samples were analyzed in a FACScalibur Flow Cytometer (BD Biosciences), and fluorescence intensity was calculated using FCAP Array v3 Software (BD Biosciences). All four cytokines exhibited single wellseparated peaks. Four individual cytokine standard curves (range $20-5000 \mathrm{pg} / \mathrm{mL}$ ) were run in each assay. Cytokine concentrations were determined by reference to standard curves and expressed in $\mathrm{pg} / \mathrm{mL}$.

2.7. Histology. Heart and skeletal muscle samples were aseptically isolated, rinsed with sterile PBS, and fixed in $4 \%$ paraformaldehyde in PBS (pH 7.4) for 24h. Fixed samples were embedded in paraffin, sectioned $(5 \mu \mathrm{m})$, stained with hematoxylin and eosin or Masson trichrome, and examined by light microscopy (Nikon Eclipse E600). To examine inflammatory infiltrate/myocarditis or myositis and amastigote 
nets, each tissue section was analyzed for $>10$ microscopic fields (40x magnifications) by two investigators who were blinded to identity of the experimental groups. Myocarditis or myositis (presence of inflammatory cells) from $\mathrm{H} \& \mathrm{E}-$ stained sections was scored as 0 (absent, without foci of inflammation), 1 (1 or less foci of inflammatory cells/field), 2 (moderate, $>2$ foci/field), 3 (generalized coalescing of foci of inflammation or disseminated inflammation with minimal cell necrosis and retention of tissue integrity), and 4 (diffused inflammation with severe tissue necrosis, interstitial edema, and loss of integrity).

2.8. Statistical Analysis. The results were expressed as the means \pm SD. Statistical analysis was performed using oneway ANOVA followed by Tukey's test. The survival time was calculated by the Kaplan-Meier method. Data sets that were found not to be normally distributed were analyzed with the Kruskal-Wallis test followed by the Mann-Whitney test to assess the differences between pairwise comparisons. Differences were considered to be statistically significant when the $p$ value was $<0.01$ or $<0.05$.

\section{Results}

3.1. Polyclonal Antibodies Anti-TcENO Are Specific. In our previous work, we cloned the enolase gene sequence into the pRSETB vector and purified the recombinant $r$ TcENO protein [36]. In this report, we immunized mice with the recombinant $\mathrm{rTcENO}$ protein to produce polyclonal antibodies. The endotoxin level of rTcENO used for immunization was found to be $<1 \mathrm{EU} / \mathrm{mL}$. The serum of these immunized mice was used in western blot assays as a first antibody against $T$. cruzi total protein extract, resulting in a specific band of approximately $46 \mathrm{kDa}$. This band was also detected when anti-pBKTcENO antibodies were used (Figure 1(a)). To confirm the specificity of the antibodies generated by immunization with rTcENO and pBKTcENO, the antibodies produced were used in western blot assays against purified rTcENO. Both antibodies recognized the recombinant protein, indicating that the antibodies generated were specific (Figure 1(b)).

The antibodies against rTcENO were also used in immunofluorescence assays against permeabilized and nonpermeabilized T. cruzi cells. In permeabilized parasites, TcENO labeling was found in the cytoplasm, and no reaction was detected in structures such as the flagellum, whereas in nonpermeabilized parasites, labeling was found in the membrane. This localization might facilitate its recognition by the host's immune system, suggesting that TcENO could have strong antigenic properties (Figure 1(c)).

3.2. Humoral Response in Mice Immunized with $r$ TcENO or pBKTcENO. To characterize the immune response induced by T. cruzi enolase, we immunized BALB/c mice intraperitoneally with rTcENO or intramuscularly with pBKTcENO as described in Materials and Methods. The immunizations with either rTcENO or pBKTcENO induced a significant production of IgGs against the rTcENO antigen (Figure 2) seven days after the last immunization. Moreover, antigen- specific isotypes of immunoglobulins in the sera of immunized animals with rTcENO revealed high levels of IgG1 > IgG2b $>\operatorname{IgG} 2 \mathrm{a}$ (Figure 3(a)), indicating that this antigen induced a mixed Th1-/Th2-like immune response. In contrast, the mice immunized with pBKTcENO showed IgG2a $>\operatorname{IgG} 2 \mathrm{~b}>\operatorname{IgG} 1$ with an IgG2b/IgG1 ratio > 1, suggesting that a predominantly Th1-like immune response was induced (Figure 3(b)). As expected, the mice immunized with rTcENO or pBKTcENO exhibited high titers of antibodies compared to the control groups inoculated with $\mathrm{PBS}$ or $\mathrm{pBK}-\mathrm{CMV}(p<0.01)$. In contrast, the isotyping mice immunized with $\mathrm{pBKT}$ CENO did not show any significant difference from those in the $\mathrm{pBK}-\mathrm{CMV}$ group.

3.3. $r T c E N O$ or $P B K T c E N O$ Immunizations Reduce the Parasitemia, but Only Mice Immunized with rTcENO Survive after Challenge with T. cruzi. To determine if immunizations with either $\mathrm{rTcENO}$ or $\mathrm{pBKTcENO}$ reduce parasitic burden in the blood and confer protection to experimentally infected mice with $T$. cruzi, we recorded parasitemia profiles and mortality rates. The parasitemia profile showed a $69.8 \%$ decrease when the mice were immunized with rTcENO compared to the control group (PBS) $(p<0.01) \quad$ (Figure $4(\mathrm{a}))$. The pBKTcENO plasmid also reduced the parasite load in the mice at $70 \%$ and $42 \%$ during the parasitemia peak (day 24 after challenge) compared to the PBS and pBK-CMV controls, respectively $(p<0.01)$.

The mice immunized with rTcENO showed $75 \%$ survival rate compared to the control group (PBS) $(p<0.01)$ at the end of the experiment. Nevertheless, all mice immunized with $\mathrm{pBKT}$ CENO eventually died, not exceeding day 33 postinfection (Figure 4(b)).

\subsection{Th1-Type Immune Response Is Polarized in rTcENO-} Immunized Mice after Parasite Challenge. To determine the type of immunological response induced after parasite challenge, the mice were bled after the peak of parasitemia (day 30 after challenge), and serum IFN- $\gamma$, IL-2, TNF, and IL-4 cytokines were determined (Figure 5). The mice vaccinated with rTcENO showed increased levels of the Th1related cytokines IFN- $\gamma$ and IL-2 but low levels of TNF compared with the nonvaccinated control group $(p<0.01)$. In contrast, the mice vaccinated with pBKTcENO showed significantly higher levels of TNF and IFN- $\gamma$ and lower levels of IL-2, but these latter levels were significantly higher than those found in nonvaccinated mice. The levels of TNF in the control mice vaccinated with the plasmid pBK-CMV were higher than those in the mice vaccinated with pBKTcENO $(p<0.01)$.

3.5. Immunization with rTcENO Confers Protection to the Heart and Skeletal Muscle. Histological analysis of the heart of nonvaccinated mice and that of mice immunized with $\mathrm{pBK}-\mathrm{CMV}$ revealed histopathological features compatible with acute chagasic myocarditis. We observed numerous nests of $T$. cruzi amastigotes accompanied by inflammatory infiltrates and few affected myocardial fibers that showed regeneration. In contrast, histological analysis of the heart of mice immunized with $\mathrm{rTcENO}$ revealed 

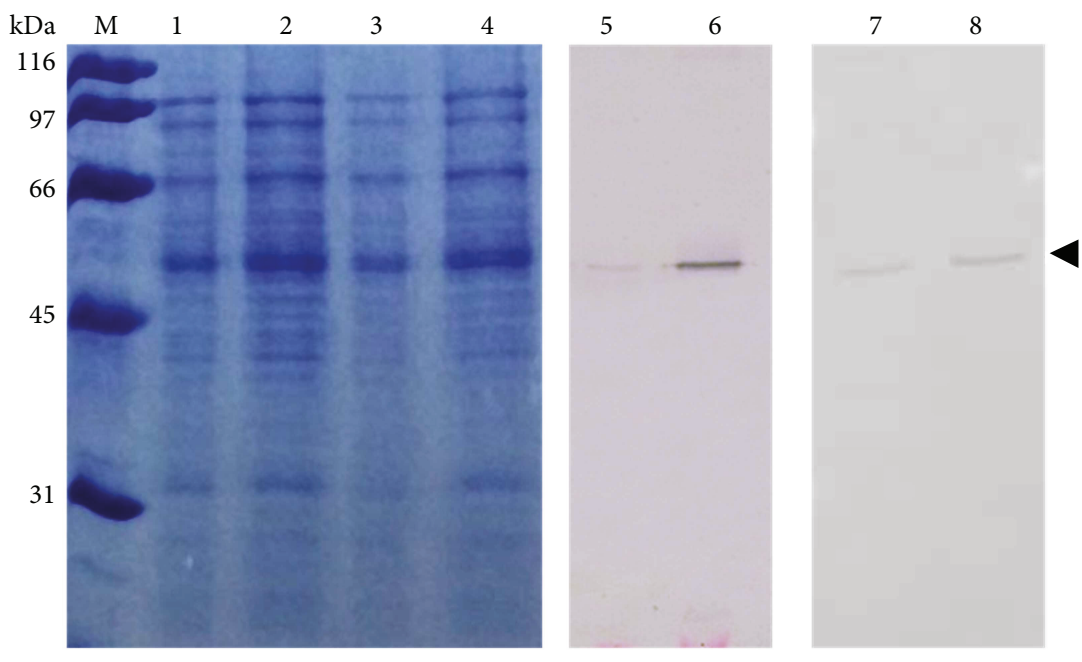

(a)

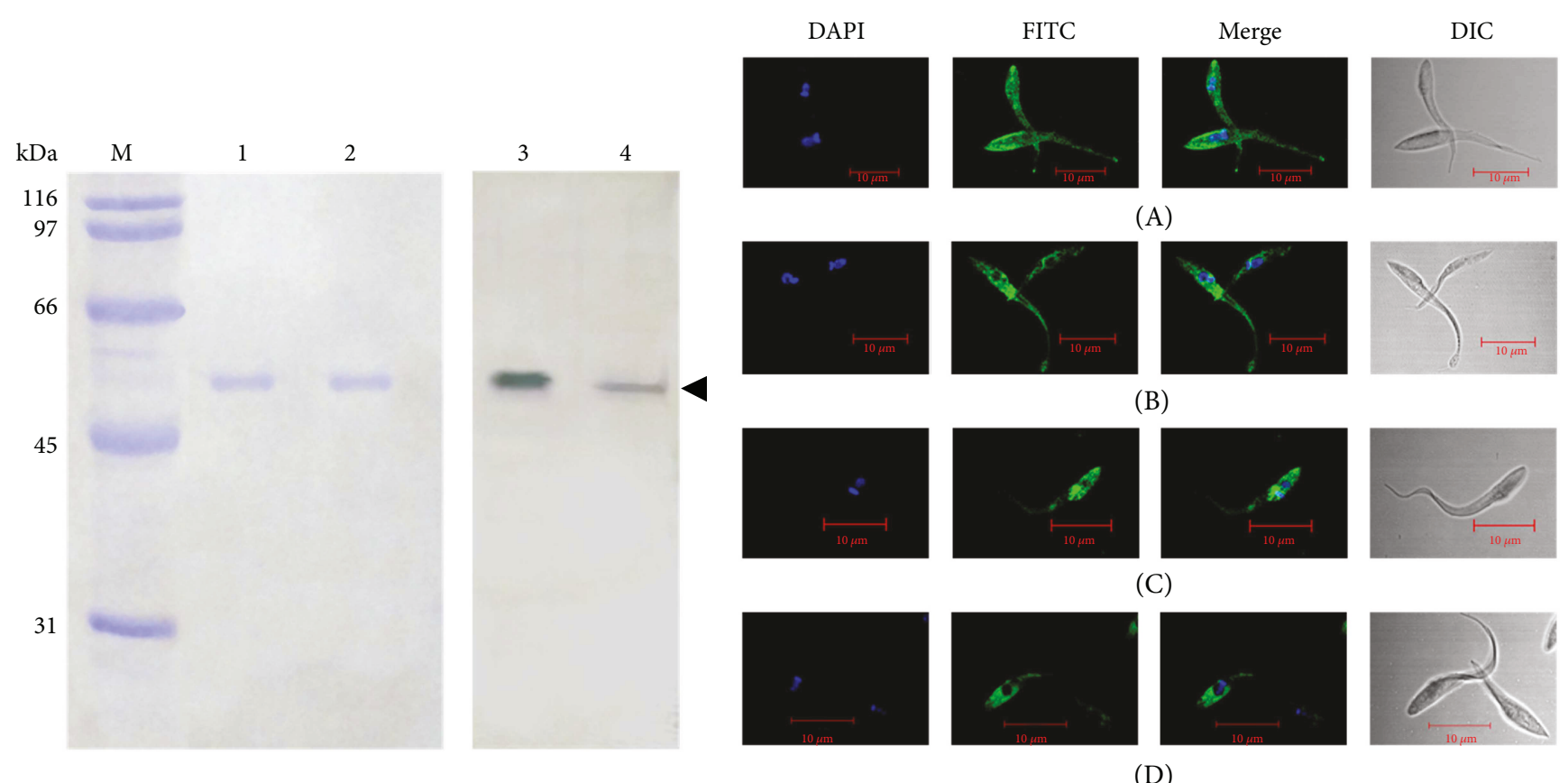

(b)

(c)

FIgure 1: T. cruzi enolase immunodetection. (a) Total extract of T. cruzi soluble proteins and its respective replica for western blotting. Lane M: molecular weight marker; lanes 1 and 3: total soluble proteins ( $10 \mu \mathrm{g})$; lanes 2 and 4: total soluble proteins (20 $\mu \mathrm{g})$; lanes 5 and 6: western blot of immobilized proteins with polyclonal antibodies anti-rTcENO; lanes 7 and 8: western blot of immobilized proteins with polyclonal antibodies anti-pBKTcENO. (b) Purified rTcENO and its respective replica for western blotting. Lane M: molecular weight marker; lanes 1 and 2: rTcENO $(10 \mu \mathrm{g})$; lane 3: western blot with pool of polyclonal antibodies anti-rTcENO; lane 4: western blot with pool of polyclonal antibodies anti-pBKTcENO. The arrowhead indicates the signal for a band of approximately $46 \mathrm{kDa}$ (the TcENO estimated weight). (c) Indirect immunofluorescence assay. The secondary antibody that recognized the anti-rTcEno was FITC labeled (green fluorescence). Nuclear and kinetoplast DNA were stained with DAPI and shown by blue fluorescence. Each image is a representative of at least two independent experiments and captured by confocal microscope. (A) Nonpermeabilized parasite with anti-T. cruzi polyclonal antibodies; (B) nonpermeabilized parasite with anti-rTcENO polyclonal antibodies; (C) permeabilized parasite with anti-T. cruzi polyclonal antibodies; (D) permeabilized parasite with anti-rTcENO polyclonal antibodies.

diffuse myocarditis with a mild mononuclear inflammatory infiltrate composed mainly of lymphocytes and a few plasma cells, while amastigote nests were absent. In mice immunized with $\mathrm{pBKTCENO}$, severe myocarditis, with the presence of amastigote nest associated with necrosis of the myocardial fibers, and intense inflammatory infiltrates, composed of lymphocytes, plasma cells, and a few polymorphonuclear leukocytes, were observed (Figure 6).

Skeletal muscle samples of the control mice exhibited disorganization of tissue architecture, necrosis, and large nests of T. cruzi amastigotes, while in the vaccinated mice, the skeletal muscle showed inflammatory infiltrates that consisted 


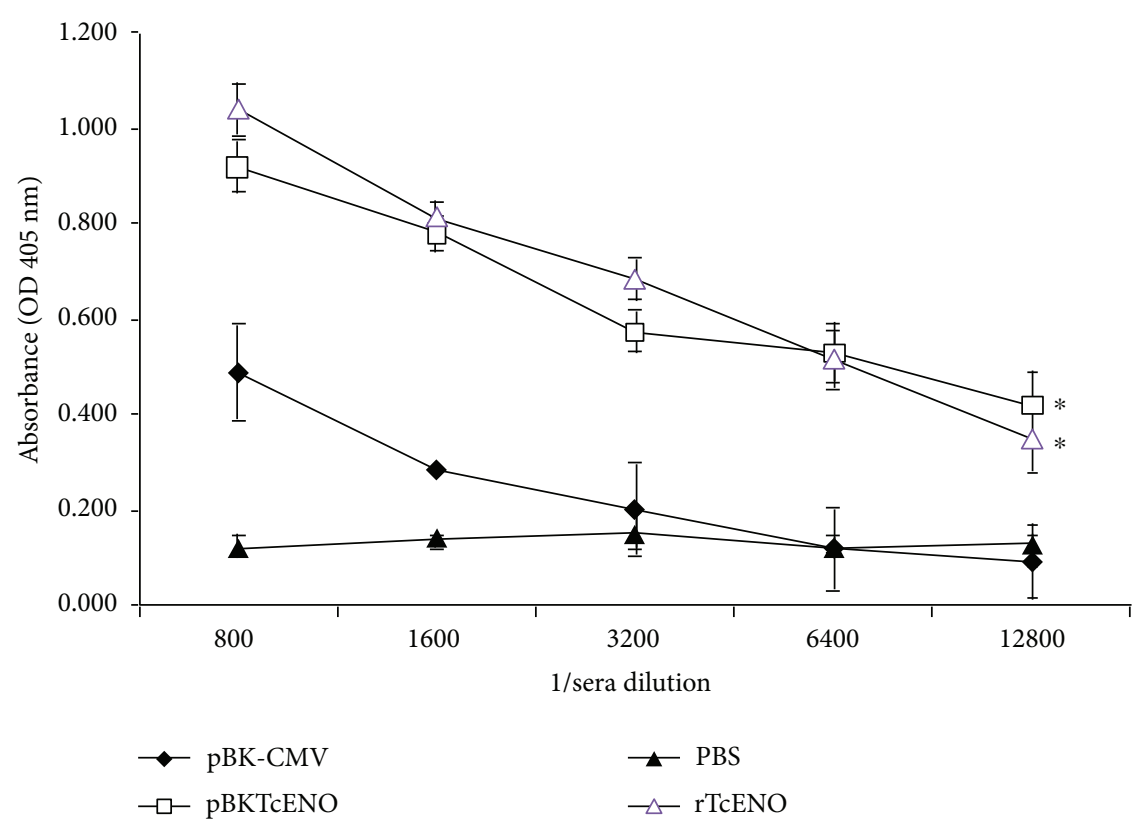

FIgURE 2: Antibody titers in immunized mice. ELISA was performed seven days after the last immunization to evaluate the serum levels (absorbance in optical density at $405 \mathrm{~nm}$ ) of rTcENO-specific antibodies at different dilutions. The values represent the average of triplicate assays \pm S.D. A significant difference was detected by comparing rTcENO or $\mathrm{pBKTcENO}$ versus $\mathrm{PBS}$ or $\mathrm{pBK}-\mathrm{CMV}\left({ }^{*} p<0.01\right)$.

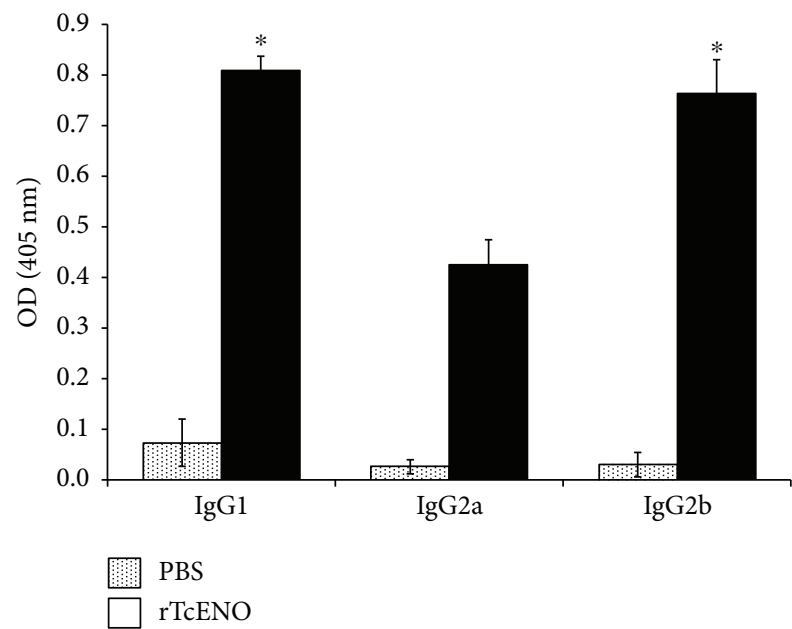

(a)

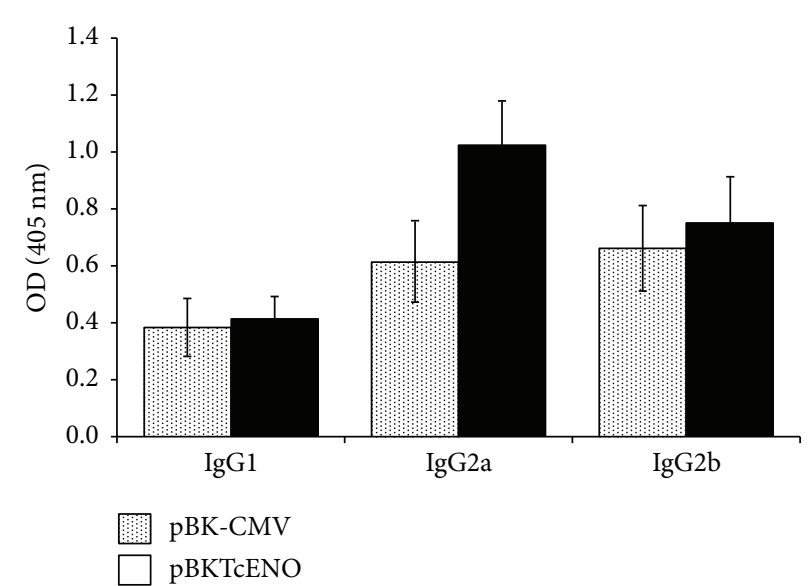

(b)

FIgure 3: Antibody isotypes in immunized mice. Seven days after the last immunization with rTcENO (a) or pBKTcENO (b), generated antibody isotypes were evaluated by ELISA. The plotted data show optical density (OD) values for eight mice per group representing the mean $\pm S D$ of at least three independent experiments. A significant difference was detected in immunized mice with $\mathrm{rTcENO}$ by comparing IgG1 and IgG2b versus IgG2a $\left({ }^{*} p<0.01\right)$.

mainly of mononuclear cells. We did not detect any amastigote nests in the mice vaccinated with rTcENO (Figure 7).

\section{Discussion}

Chagas disease is a neglected tropical disease that affects the poorest population in endemic areas. Recently, several forms of transmission have reemerged and affected the population in nonendemic countries $[9,44]$. Specific treatment of this disease consists of two drugs, nifurtimox and benznidazole. The efficacy of both drugs in the acute phase has been shown; however, their use in the chronic phase is currently the subject of discussion. Unfortunately, a vaccine for Chagas disease is not available to date $[11,45]$.

DNA vaccination has been shown to generate both humoral and cell-mediated immune responses [46] and has been shown to be an effective means of generating protective responses against $T$. cruzi infection in murine models [15, 42, 47]. Moreover, immunization with recombinant proteins has also generated promising results $[16,19$, $48,49]$. Several membrane proteins have been proposed as good options to develop vaccines against different diseases. 


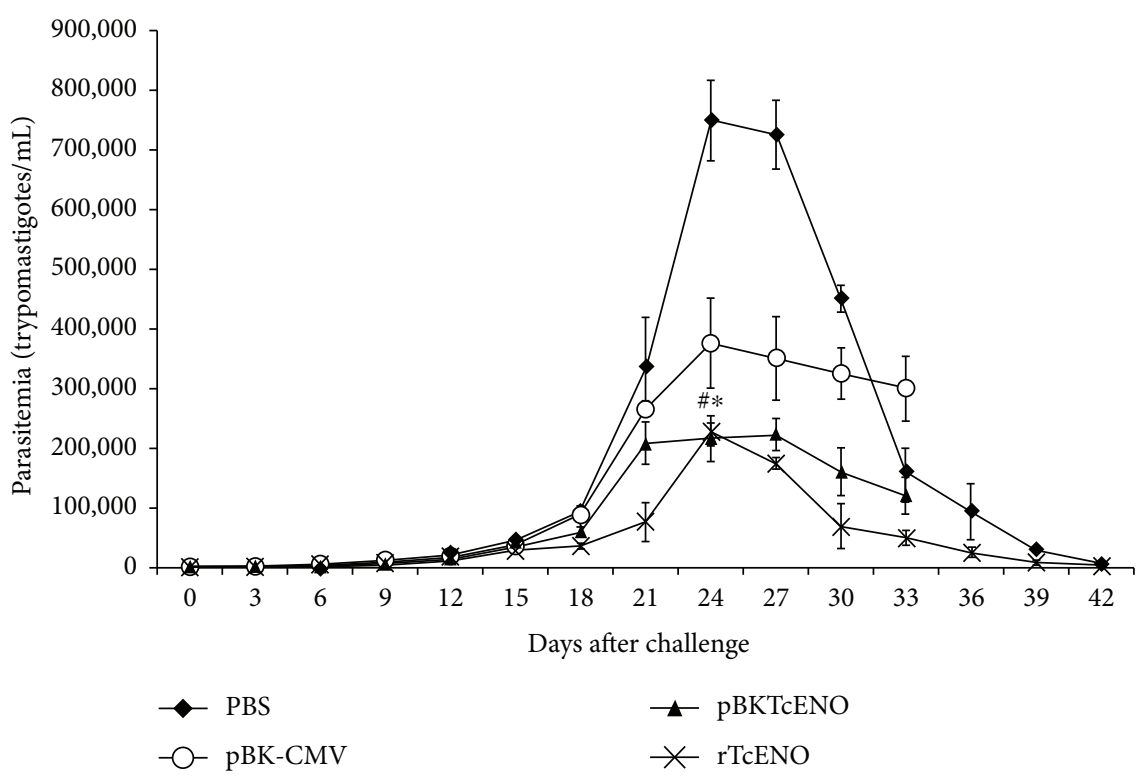

(a)

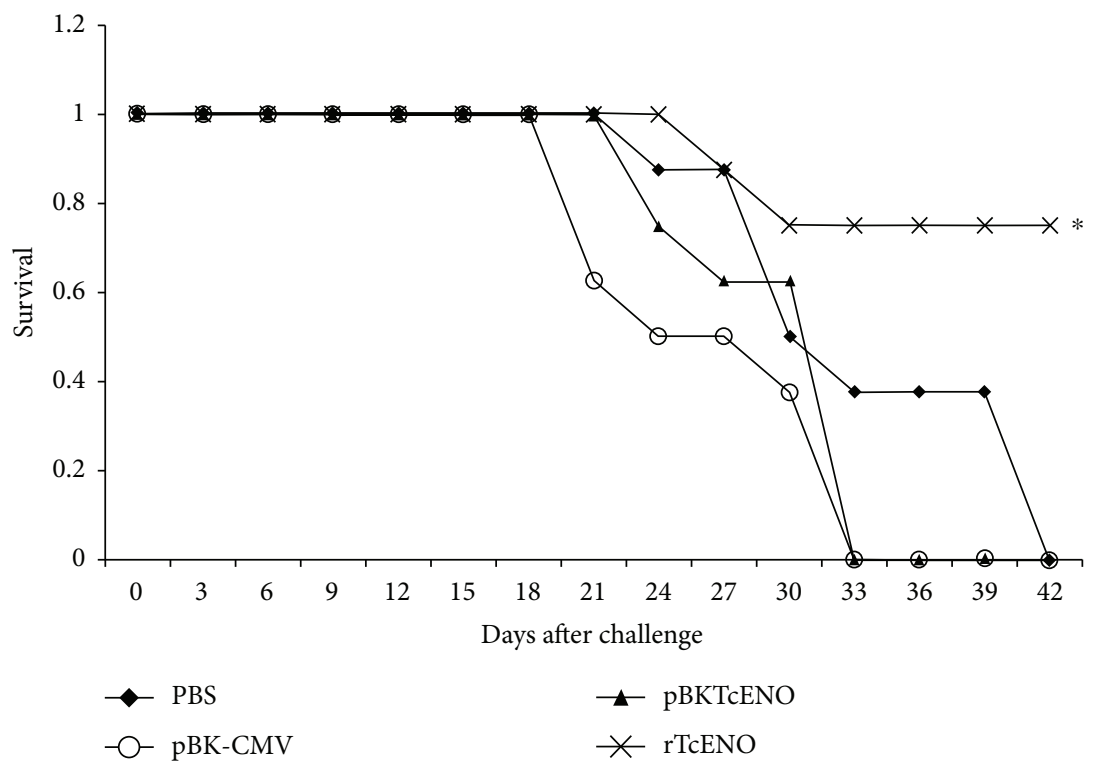

(b)

FIGURE 4: Evaluation of protection generated by vaccination with rTcENO or pBKTcENO. (a) Parasitemia in immunized mice after challenge with T. cruzi. BALB/c mice were immunized as described in Materials and Methods. Values plotted show the mean \pm standard deviation of eight mice per group and are representative of two independent experiments. At the peak of infection, parasitemia levels were compared using one-way analysis of variance and post hoc Tukey's tests. A significant difference was detected by comparing rTcENO versus PBS $\left({ }^{*} p<0.01\right)$, pBKTcENO versus PBS $\left({ }^{\#} p<0.01\right)$ and pBKTcENO versus pBK-CMV $\left({ }^{*} p<0.01\right)$. (b) Survival of immunized and infected mice. Values plotted show the mean \pm SD of eight mice per group and are representative of two independent experiments with similar results. A statistically significant difference $\left({ }^{*} p<0.01\right)$ between $\mathrm{rTcENO}$ versus PBS is indicated.

Among these membrane proteins, enolase has been demonstrated to be immunogenic [20-22] suggesting that a vaccine with this protein is possible. In our previous work, we evaluated the immunogenic characteristics of T. cruzi enolase using in silico assays. Our study showed that the recombinant protein was recognized by serum from both mice and humans infected with T. cruzi [36]. To support these data, in this study, we used rTcEno polyclonal antibodies against total protein extracts of $T$. cruzi in western blot and immunofluorescence assays to determine the specificity of these antibodies. These results demonstrated that enolase was located in both the cytoplasm and the cell membrane, in agreement with studies carried out in parasites such as Echinococcus granulosus, Trichomonas vaginalis, and Plasmodium falciparum [20, 50, 51].

Antibodies against both rTcENO and pBKTcENO were bound to intact epimastigotes, indicating that enolase protein was antigenic and exposed on the parasite surface. 


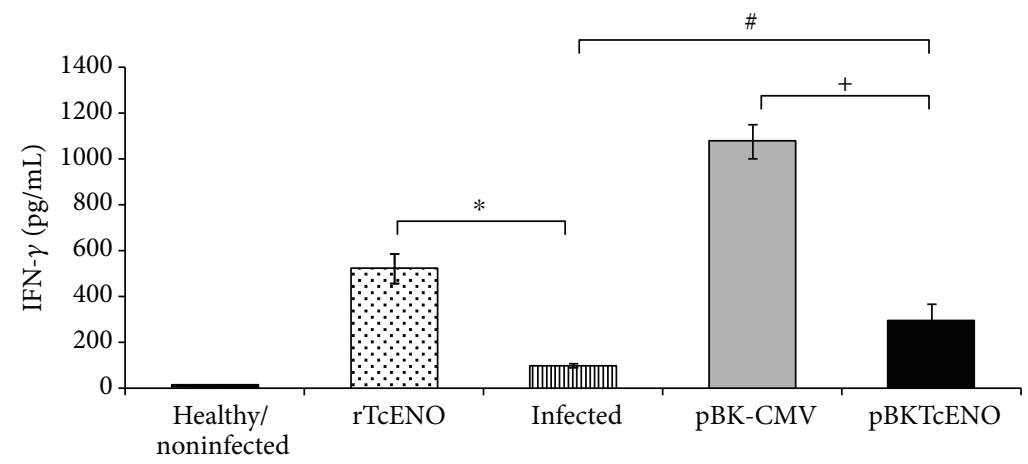

(a)

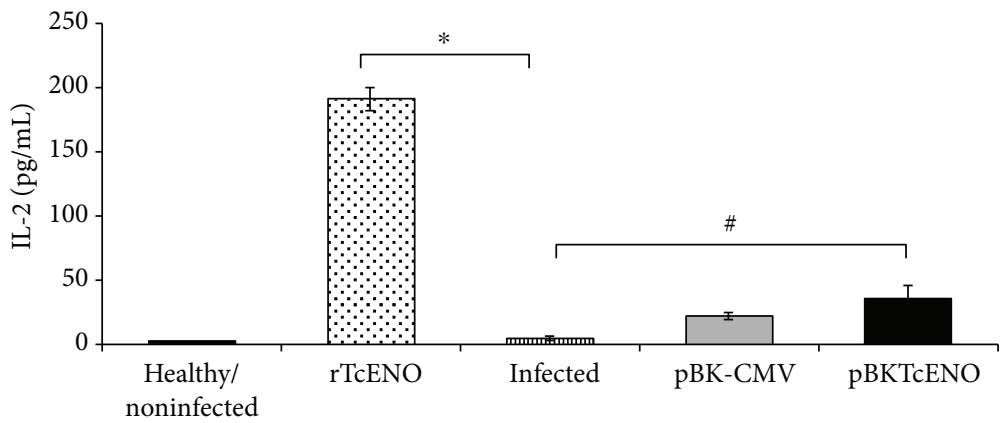

(b)

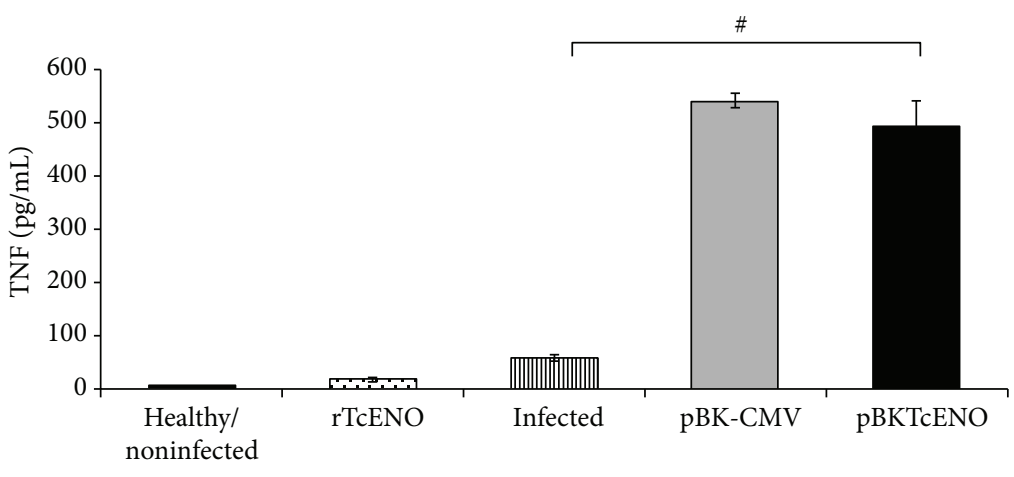

(c)

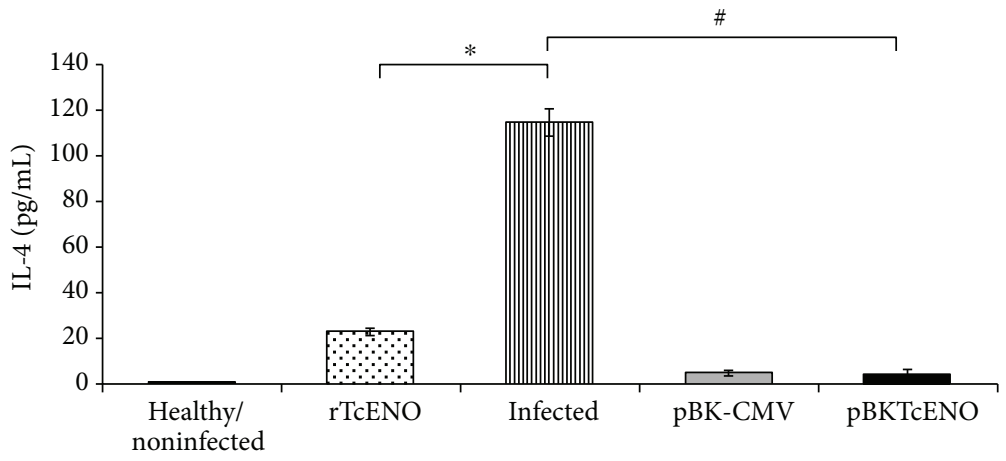

(d)

FIGURE 5: Serum cytokine levels in immunized and nonimmunized mice after challenge. Serum levels of (a) IFN- $\gamma$, (b) IL-2, (c) TNF, and (d) IL-4 were determined by flow cytometry. The concentration of a particular cytokine was established by comparing the obtained data with a standard curve for each experiment. The data are represented as the mean \pm SD. All measurements were performed in duplicate, with serum from eight mice per group. Sera from healthy mice (noninfected and nonimmunized) were used as controls. Significant differences were detected as follows: rTcENO versus infected $\left({ }^{*} p<0.01\right)$, pBKTcENO versus infected $\left({ }^{\#} p<0.01\right)$, and pBKTcENO versus pBK-CMV $\left({ }^{+} p<0.01\right)$. 


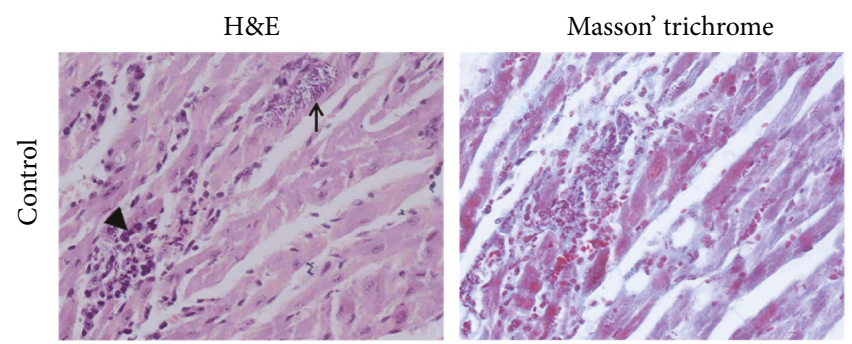

(A)

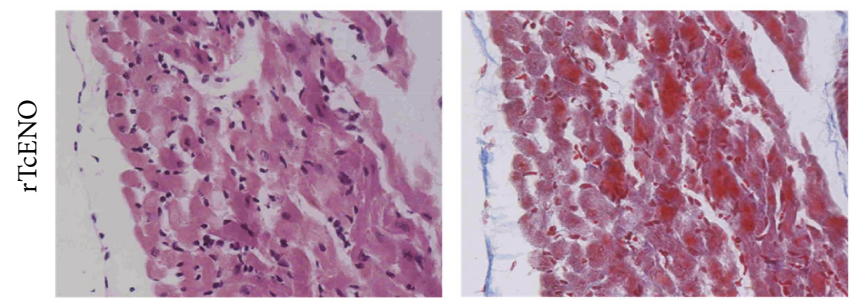

(B)

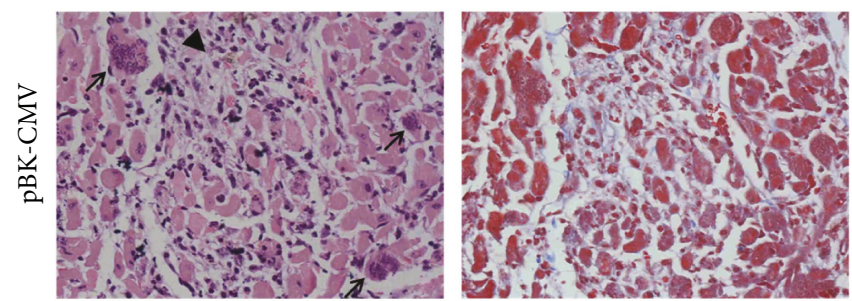

(C)

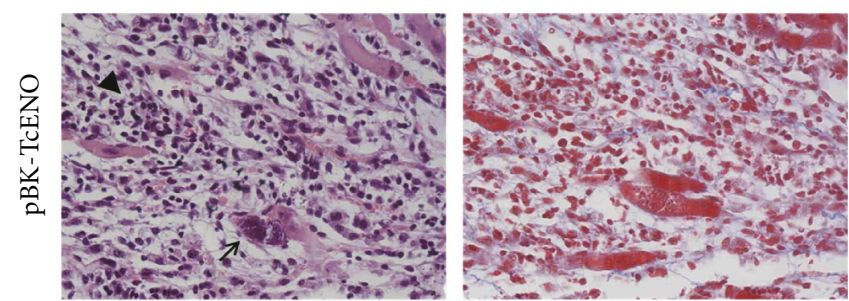

(D)

(a)

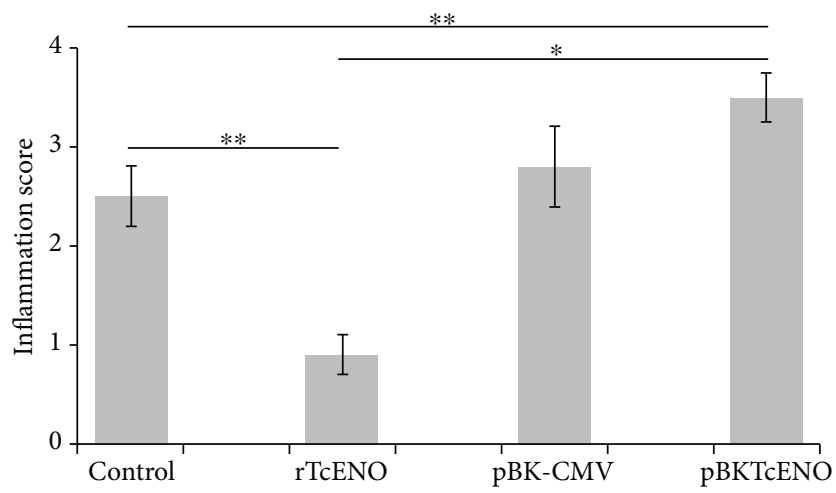

(b)

Figure 6: Vaccination effects on the cardiac muscle sections. (a) Tissue sections were stained with hematoxylin and eosin or Masson's trichrome and visualized by light microscopy (original magnification: 40x). Representative micrographs of the heart tissue from controlinfected mice and mock-immunized with PBS (A), rTcENO (B), pBK-CMV empty plasmid vector (C), and pBK-TcENO (D) are shown. Black arrows show amastigote nests. Black arrowheads show inflammatory infiltrates. (b) Inflammatory lesion (lymphocyte infiltrates) scores. The inflammatory score was derived from two different experiments as described in Materials and Methods. The data are expressed as mean $\pm \mathrm{SD}$, and significance is presented as ${ }^{*} p<0.01$ and ${ }^{* *} p<0.05\left({ }^{* *}\right.$ control group nonimmunized and infected versus vaccinated and infected groups, rTcENO and pBK-TcENO; * rTcENO versus pBK-TcENO). 


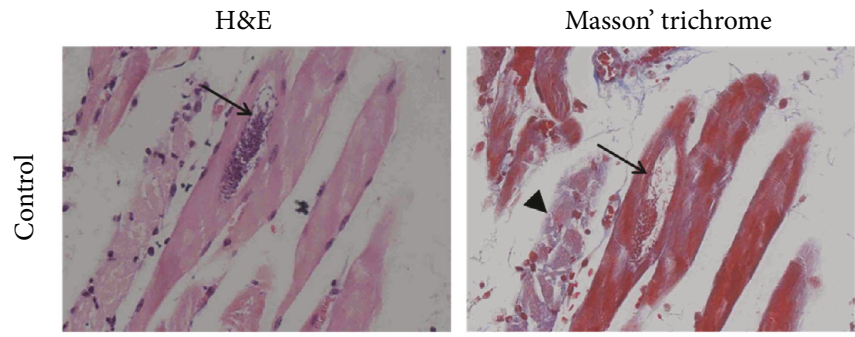

(A)

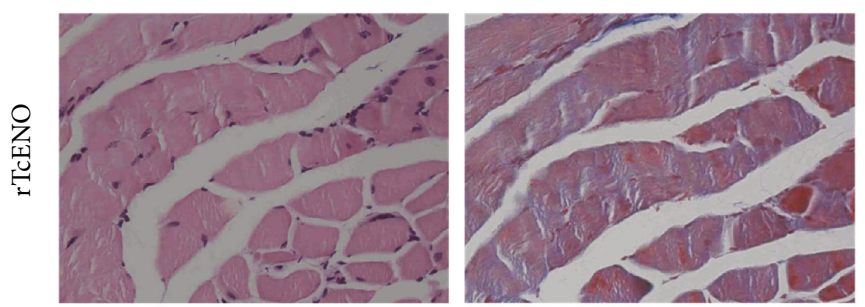

(B)

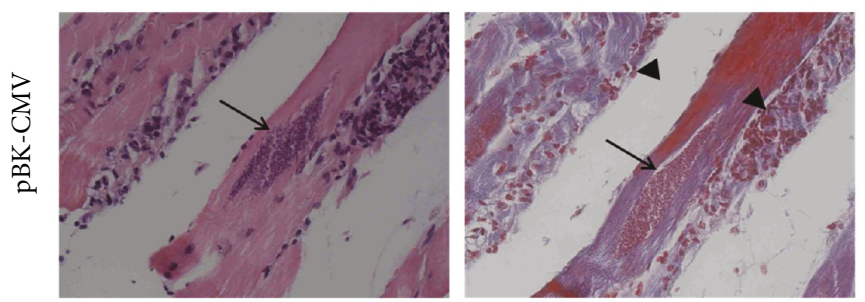

(C)

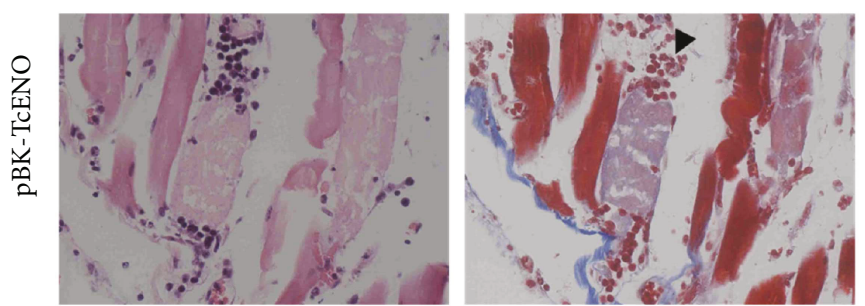

(D)

(a)

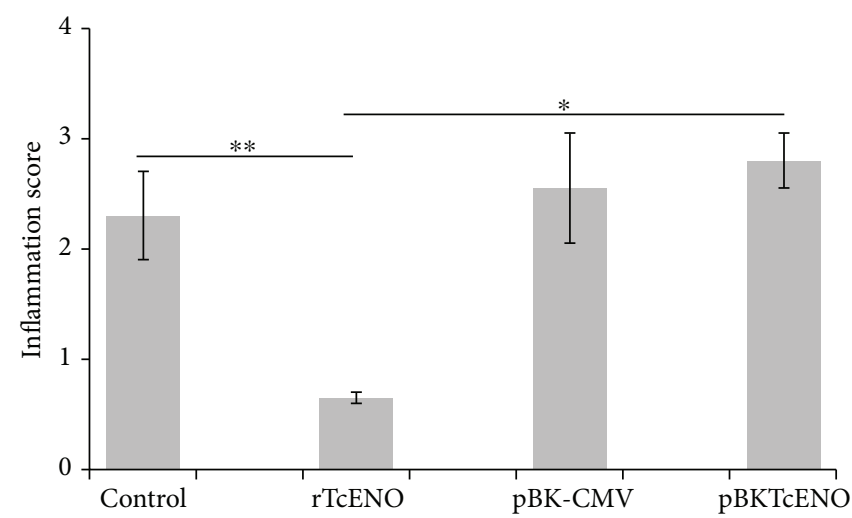

(b)

Figure 7: Vaccination effects on the skeletal muscle sections. (a) Tissue sections were stained with hematoxylin and eosin or Masson's trichrome and visualized by light microscopy (original magnification: 40x). Representative micrographs of skeletal tissue from controlinfected mice and mock-immunized with PBS (A), rTcENO (B), pBK-CMV empty plasmid vector (C), and pBK-TcENO (D) are shown. Black arrows show amastigote nests. Black arrowheads show inflammatory infiltrates. (b) Inflammatory lesion (lymphocyte infiltrates) scores. The inflammatory score was derived from two different experiments as described in Materials and Methods. The data are expressed as mean $\pm \mathrm{SD}$, and significance is presented as ${ }^{*} p<0.01$ and ${ }^{* *} p<0.05\left({ }^{* *}\right.$ control group nonimmunized and infected versus vaccinated and infected groups, rTcENO and pBK-TcENO; * rTcENO versus pBK-TcENO). 
Nevertheless, the exact mechanism by which enolase is secreted and translocated to the parasite surface is still unknown. However, it is known that the amino acid sequence lacks a conventional $\mathrm{N}$-terminal signal sequence. It has been suggested that enolases could be secreted in exosomes and other vesicles [52] and that secreted enolases could reassociate with the cell membrane [24].

Enolase has been analyzed as a vaccine in several parasites and bacteria, demonstrating that it may be an important immunogenic protein and a protective antigen [20-22, 5355]. In this study, analysis of the humoral immune response showed that the generated antibodies in the mice vaccinated with rTcENO were a mixture of Th1- and Th2-type immune responses (IgG1 $>\operatorname{IgG} 2 \mathrm{~b}>\operatorname{IgG} 2 \mathrm{a})$. Moreover, the mice vaccinated with pBKTcENO showed an increase in IgG2a immune response and the rate of IgG2b/IgG1 being $>1$, which suggested the dominance of Th1-type immune response. Previous studies showed that Th1-type immune response was required for clearing the parasite from infected mice $[56,57]$.

We found that the mice immunized with rTcENO were capable of significantly inducing IL- 2 and IFN- $\gamma$ in comparison to the control group, showing that in the immune response after challenge, there was a polarization towards Th1-type immune response. In the mice vaccinated with pBKTcENO, there was induction of IFN $-\gamma$ and TNF but not IL-2. IFN- $\gamma$ was required to activate macrophages and indirectly constitutes an important source of protective proinflammatory cytokines, which could effectively kill intracellular parasites such as $T$. cruzi by nitric oxide(NO-) dependent mechanisms [58]. In this experimental group, discrete levels of IFN- $\gamma$ were detected, and animals showed reduction in parasitemia profile. However, no mice survived at day 33 postchallenge. Interestingly, the pBK$\mathrm{CMV}$-immunized animals were able to induce high levels of IFN- $\gamma$ and decrease parasitemia significantly compared to the PBS control group. Despite its low parasitemia, this group also showed reduced survival. These results might be due to the induced response by immunostimulatory sequences in the plasmid that trigger innate immunity in the host [59] but could not confer a specific response that favors the survival of the animals against parasitic challenge.

Therefore, other factors that did not prevent mortality might be involved in the reduction of parasitemia. One of these factors could be the IL-10 cytokine because it acted as an immunoregulatory cytokine in the Th1-type response [60]. In turn, IL-10 would prevent the collateral damage generated by a strong immune response against the parasite and suppress the development of inflammatory cell infiltrates that otherwise would be exacerbated. A previous study proposed that an exacerbated response to infections could result in harmful injuries [61].

To determine whether vaccination conferred protection in the cardiac and skeletal muscle, histological sections were prepared and stained. The results showed that animals immunized with rTcENO were able to prevent the establishment of parasites in the heart tissue, as shown by the absence of amastigote nests and low amount of inflammatory infiltrates in the heart sections (Figures 6 and 7).
Furthermore, the low amount of inflammatory cells indicated that the immune response was adequate to eliminate the parasites.

Although the pBKTcENO vaccine reduced the parasitemia in the immunized/infected mice, these animals showed exacerbated damage in the heart and skeletal tissues (Figures 5 and 6), with many amastigote nests and inflammatory cells. The tissue injured by T. cruzi typically shows mononuclear cells and progression from multifocal to diffuse lesions. Activated neutrophils and eosinophils are efficient cells to destroy T. cruzi, and indirectly their activity causes severe damage to other host cells. Although neutrophils and eosinophils are not as abundant as mononuclear cells, their presence correlates with the severity of lesions observed in the cardiac tissue $[62,63]$.

Several researchers have demonstrated that DNA immunization is effective in protecting animals against $T$. cruzi, particularly with TSA-1 [64], Tc2 [65], and SSP4 [66] genes, where immunizations generated high levels of cytokines which modulate the Th1-type immune response such as IFN- $\gamma$, and in the case of SSP4, the immunization also favored the increase of IL- 10 .

The exact mechanism by which pBKTcENO-immunized/ infected or pBK-CMV-mock-immunized/infected mice died after challenge with $T$. cruzi even after producing antibodies related to Th1-type immune response is not known. It is possible that these antibodies were not opsonic, failing to induce T. cruzi killing by mouse phagocytes, as it was reported by Esgleas et al. [67], who found that the immunization with SsEno of Streptococcus suis failed to protect mice after experimental infection with bacteria. Another possible explanation is that the immune response was exacerbated enough in the cardiac tissue to induce death of the immunized animals. In this case, IL-10 is an important pleiotropic immunoregulatory cytokine that can participate during the Th1 immune response [60] to prevent collateral damage generated by a strong immune response against the parasites and suppress the development of inflammatory cell infiltrates that otherwise would be exacerbated. However, IL-10 can suppress immune responses [68] thus favoring the observed increased levels of parasites after acute infection. Therefore, we will conduct experiments using anti-IL-10 mAbs or IL-10 KO mice to determine the role of IL-10 in the protection against death of vaccinated mice with pBKTcENO.

In summary, we have shown that the immunization with rTcENO effectively controlled acute T. cruzi infection in a murine model by reducing parasite burden in the blood, preventing the establishment of inflammatory infiltrates in the heart and skeletal muscle and increasing the survival of immunized mice. Our data support further studies to improve the efficacy of rTcENO and examine its potential as a good candidate for the development of a vaccine against T. cruzi.

\section{Data Availability}

The data used to support the findings of this study are available from the corresponding author upon request. 


\section{Disclosure}

María Cristina González-Vázquez’s and Alejandro CarabarinLima's current address is Instituto de Ciencias, Benemérita Universidad Autónoma de Puebla, Edificio IC10, Ciudad Universitaria, Col. San Manuel, 72570 Puebla, PUE, Mexico.

\section{Conflicts of Interest}

No potential conflicts of interest were disclosed.

\section{Acknowledgments}

The authors are grateful to Sandra Aguilar Pérez and Benito Salvador Chávez Rentería for their help with tissue processing. The authors thank Lidia Baylón Pacheco and Enrique Martínez de Luna for their technical help. Alejandro Carabarin-Lima was a recipient of a postdoctoral fellowship from the ICyTDF, Mexico. This work was supported by a grant from the Consejo Nacional de Ciencia y Tecnología (CONACyT), Mexico (Grant no. 69081FONSECSSA/IMSS/ ISSSTE).

\section{References}

[1] WHO, "World Health Organization," 2017, March 2018, http://www.who.int/chagas/en/.

[2] J. A. Perez-Molina and I. Molina, "Chagas disease," Lancet, vol. 391, no. 10115, pp. 82-94, 2018.

[3] World Health Organization, Chagas Disease (American Trypanosomiasis), World Health Organization, Geneva, 2015, March 2018, http://www.who.int/mediacentre/factsheets/ fs340/en/.

[4] C. Bern, S. Kjos, M. J. Yabsley, and S. P. Montgomery, "Trypanosoma cruzi and Chagas' disease in the United States," Clinical Microbiology Reviews, vol. 24, no. 4, pp. 655681, 2011.

[5] Y. Jackson, L. Gétaz, H. Wolff et al., "Prevalence, clinical staging and risk for blood-borne transmission of Chagas disease among Latin American migrants in Geneva, Switzerland," PLoS Neglected Tropical Diseases, vol. 4, no. 2, article e592, 2010.

[6] F. X. Lescure, A. Canestri, H. Melliez et al., "Chagas disease, France," Emerging Infectious Diseases, vol. 14, no. 4, pp. 644646, 2008.

[7] J. Muñoz, O. Coll, T. Juncosa et al., "Prevalence and vertical transmission of Trypanosoma cruzi infection among pregnant Latin American women attending 2 maternity clinics in Barcelona, Spain," Clinical Infectious Diseases, vol. 48, no. 12, pp. 1736-1740, 2009.

[8] G. A. Schmunis, "Epidemiology of Chagas disease in non endemic countries: the role of international migration," Memórias do Instituto Oswaldo Cruz, vol. 102, Supplement 1, pp. 75-86, 2007.

[9] A. Carabarin-Lima, M. C. González-Vázquez, O. RodríguezMorales et al., "Chagas disease (American trypanosomiasis) in Mexico: an update," Acta Tropica, vol. 127, no. 2, pp. 126$135,2013$.

[10] F. J. Carod-Artal and J. Gascon, "Chagas disease and stroke," Lancet Neurology, vol. 9, no. 5, pp. 533-542, 2010.
[11] L. M. G. Bahia-Oliveira, J. A. S. Gomes, J. R. Cançado et al., "Immunological and clinical evaluation of chagasic patients subjected to chemotherapy during the acute phase of Trypanosoma cruzi infection 14-30 years ago," The Journal of Infectious Diseases, vol. 182, no. 2, pp. 634-638, 2000.

[12] J. R. Cancado, "Criteria of Chagas disease cure," Memórias do Instituto Oswaldo Cruz, vol. 94, Supplement 1, pp. 331-335, 1999.

[13] S. I. Cazorla, P. D. Becker, F. M. Frank et al., "Oral vaccination with Salmonella enterica as a cruzipain-DNA delivery system confers protective immunity against Trypanosoma cruzi," Infection and Immunity, vol. 76, no. 1, pp. 324333, 2008.

[14] F. M. Frank, S. I. Cazorla, M. J. Sartori, and R. S. Corral, "Elicitation of specific, Th1-biased immune response precludes skeletal muscle damage in cruzipain-vaccinated mice," Experimental and Molecular Pathology, vol. 84, no. 1, pp. 6470, 2008.

[15] F. Costa, G. Franchin, V. L. Pereira-Chioccola, M. Ribeirao, S. Schenkman, and M. M. Rodrigues, "Immunization with a plasmid DNA containing the gene of trans-sialidase reduces Trypanosoma cruzi infection in mice," Vaccine, vol. 16, no. 8, pp. 768-774, 1998.

[16] D. F. Hoft, C. S. Eickhoff, O. K. Giddings, J. R. C. Vasconcelos, and M. M. Rodrigues, "Trans-sialidase recombinant protein mixed with CpG motif-containing oligodeoxynucleotide induces protective mucosal and systemic Trypanosoma cruzi immunity involving CD8+ CTL and B cell-mediated crosspriming," Journal of Immunology, vol. 179, no. 10, pp. 68896900, 2007.

[17] E. L. V. Silveira, C. Claser, F. A. B. Haolla, L. G. Zanella, and M. M. Rodrigues, "Novel protective antigens expressed by Trypanosoma cruzi amastigotes provide immunity to mice highly susceptible to Chagas' disease," Clinical and Vaccine Immunology, vol. 15, no. 8, pp. 1292-1300, 2008.

[18] B. H. Fralish and R. L. Tarleton, "Genetic immunization with LYT1 or a pool of trans-sialidase genes protects mice from lethal Trypanosoma cruzi infection," Vaccine, vol. 21, no. 2122, pp. 3070-3080, 2003.

[19] K. A. Luhrs, D. L. Fouts, and J. E. Manning, "Immunization with recombinant paraflagellar rod protein induces protective immunity against Trypanosoma cruzi infection," Vaccine, vol. 21, no. 21-22, pp. 3058-3069, 2003.

[20] I. Pal-Bhowmick, M. Mehta, I. Coppens, S. Sharma, and G. K. Jarori, "Protective properties and surface localization of Plasmodium falciparum enolase," Infection and Immunity, vol. 75, no. 11, pp. 5500-5508, 2007.

[21] W. Q. Li, X. C. Hu, X. Zhang et al., "Immunisation with the glycolytic enzyme enolase confers effective protection against Candida albicans infection in mice," Vaccine, vol. 29, no. 33, pp. 5526-5533, 2011.

[22] N. Chen, Z. G. Yuan, M. J. Xu et al., "Ascaris suum enolase is a potential vaccine candidate against ascariasis," Vaccine, vol. 30, no. 23, pp. 3478-3482, 2012.

[23] V. Pancholi, "Multifunctional alpha-enolase: its role in diseases," Cellular and Molecular Life Sciences, vol. 58, no. 7, pp. 902-920, 2001.

[24] S. Bergmann, M. Rohde, G. S. Chhatwal, and S. Hammerschmidt, "alpha-Enolase of Streptococcus pneumoniae is a plasmin(ogen)-binding protein displayed on the bacterial cell surface," Molecular Microbiology, vol. 40, no. 6, pp. 1273-1287, 2001. 
[25] V. Pancholi and V. A. Fischetti, "alpha-enolase, a novel strong plasmin(ogen) binding protein on the surface of pathogenic streptococci," Journal of Biological Chemistry, vol. 273, no. 23, pp. 14503-14515, 1998.

[26] D. J. P. Ferguson, S. F. Parmley, and S. Tomavo, "Evidence for nuclear localisation of two stage-specific isoenzymes of enolase in Toxoplasma gondii correlates with active parasite replication," International Journal for Parasitology, vol. 32, no. 11, pp. 1399-1410, 2002.

[27] M. Labbe, M. Peroval, C. Bourdieu, F. Girard-Misguich, and P. Pery, "Eimeria tenella enolase and pyruvate kinase: a likely role in glycolysis and in others functions," International Journal for Parasitology, vol. 36, no. 14, pp. 1443-1452, 2006.

[28] T. Mouveaux, G. Oria, E. Werkmeister et al., "Nuclear glycolytic enzyme enolase of Toxoplasma gondii functions as a transcriptional regulator," PLoS One, vol. 9, no. 8, article e105820, 2014.

[29] H. Lee, Y. Guo, M. Ohta, L. Xiong, B. Stevenson, and J. K. Zhu, "LOS2, a genetic locus required for cold-responsive gene transcription encodes a bi-functional enolase," EMBO Journal, vol. 21, no. 11, pp. 2692-2702, 2002.

[30] S. Feo, D. Arcuri, E. Piddini, R. Passantino, and A. Giallongo, "ENO1 gene product binds to the c-myc promoter and acts as a transcriptional repressor: relationship with Myc promoterbinding protein 1 (MBP-1)," FEBS Letters, vol. 473, no. 1, pp. 47-52, 2000.

[31] A. Subramanian and D. M. Miller, "Structural analysis of alpha-enolase. Mapping the functional domains involved in down-regulation of the c-myc protooncogene," The Journal of Biological Chemistry, vol. 275, no. 8, pp. 5958-5965, 2000.

[32] J. C. Wilkins, K. A. Homer, and D. Beighton, "Analysis of Streptococcus mutans proteins modulated by culture under acidic conditions," Applied and Environmental Microbiology, vol. 68, no. 5, pp. 2382-2390, 2002.

[33] B. L. Decker and W. T. Wickner, "Enolase activates homotypic vacuole fusion and protein transport to the vacuole in yeast," The Journal of Biological Chemistry, vol. 281, no. 20, pp. 14523-14528, 2006.

[34] I. Brandina, J. Graham, C. Lemaitre-Guillier et al., "Enolase takes part in a macromolecular complex associated to mitochondria in yeast," Biochimica et Biophysica Acta (BBA) - Bioenergetics, vol. 1757, no. 9-10, pp. 1217-1228, 2006.

[35] N. Entelis, I. Brandina, P. Kamenski, I. A. Krasheninnikov, R. P. Martin, and I. Tarassov, "A glycolytic enzyme, enolase, is recruited as a cofactor of tRNA targeting toward mitochondria in Saccharomyces cerevisiae," Genes and Development, vol. 20, no. 12, pp. 1609-1620, 2006.

[36] A. Carabarín-Lima, O. Rodríguez-Morales, M. C. GonzálezVázquez et al., "In silico approach for the identification of immunological properties of enolase from Trypanosoma cruzi and its possible usefulness as vaccine in Chagas disease," Parasitology Research, vol. 113, no. 3, pp. 1029-1039, 2014.

[37] W. H. Lee, H. I. Choi, S. W. Hong, K. S. Kim, Y. S. Gho, and S. G. Jeon, "Vaccination with Klebsiella pneumoniae-derived extracellular vesicles protects against bacteria-induced lethality via both humoral and cellular immunity," Experimental and Molecular Medicine, vol. 47, no. 9, article e183, 2015.

[38] K. A. Pasquevich, S. M. Estein, C. G. Samartino et al., "Immunization with recombinant Brucella species outer membrane protein Omp16 or Omp19 in adjuvant induces specific CD4 + and CD8+ T cells as well as systemic and oral protection against Brucella abortus infection," Infection and Immunity, vol. 77, no. 1, pp. 436-445, 2009.

[39] C. N. Pollak, M. M. Wanke, S. M. Estein et al., "Immunization with Brucella VirB proteins reduces organ colonization in mice through a Th1-type immune response and elicits a similar immune response in dogs," Clinical and Vaccine Immunology, vol. 22, no. 3, pp. 274-281, 2015.

[40] C. Serna, J. A. Lara, S. P. Rodrigues, A. F. Marques, I. C. Almeida, and R. A. Maldonado, "A synthetic peptide from Trypanosoma cruzi mucin-like associated surface protein as candidate for a vaccine against Chagas disease," Vaccine, vol. 32, no. 28, pp. 3525-3532, 2014.

[41] Y. Shimizu, H. Takagi, T. Nakayama et al., "Intraperitoneal immunization with oligomannose-coated liposome-entrapped soluble leishmanial antigen induces antigen-specific T-helper type immune response in $\mathrm{BALB} / \mathrm{c}$ mice through uptake by peritoneal macrophages," Parasite Immunology, vol. 29, no. 5, pp. 229-239, 2007.

[42] M. Arce-Fonseca, A. Ramos-Ligonio, A. Lopez-Monteon, B. Salgado-Jimenez, P. Talamas-Rohana, and J. L. RosalesEncina, "A DNA vaccine encoding for TcSSP4 induces protection against acute and chronic infection in experimental Chagas disease," International Journal of Biological Sciences, vol. 7, no. 9, pp. 1230-1238, 2011.

[43] National Research Council (US) Committee for the Update of the Guide for the Care and Use of Laboratory Animals, Guide for the Care and Use of Laboratory Animals, National Academies Press (US), Washington (DC), 8th edition, 2011.

[44] P. Hotez, E. Ottesen, A. Fenwick, and D. Molyneux, "The neglected tropical diseases: the ancient afflictions of stigma and poverty and the prospects for their control and elimination," Advances in Experimental Medicine and Biology, vol. 582, pp. 23-33, 2006.

[45] C. Bern, "Antitrypanosomal therapy for chronic Chagas' disease," The New England Journal of Medicine, vol. 364, no. 26, pp. 2527-2534, 2011.

[46] J. J. Donnelly, B. Wahren, and M. A. Liu, "DNA vaccines: progress and challenges," Journal of Immunology, vol. 175, no. 2, pp. 633-639, 2005.

[47] N. Garg and R. L. Tarleton, "Genetic immunization elicits antigen-specific protective immune responses and decreases disease severity in Trypanosoma cruzi infection," Infection and Immunity, vol. 70, no. 10, pp. 5547-5555, 2002.

[48] A. Carabarin-Lima, M. C. González-Vázquez, B.-P. Lidia, P.-T. Rohana, and R.-E. J. Luis, "Immunization with the recombinant surface protein $\mathrm{rTcSP} 2$ alone or fused to the CHP or ATPase domain of TcHSP70 induces protection against acute Trypanosoma cruzi infection," Journal of Vaccines \& Vaccination, vol. 1, no. 3, 2010.

[49] R. A. Wrightsman and J. E. Manning, "Paraflagellar rod proteins administered with alum and IL-12 or recombinant adenovirus expressing IL-12 generates antigen-specific responses and protective immunity in mice against Trypanosoma cruzi," Vaccine, vol. 18, no. 14, pp. 1419-1427, 2000.

[50] W. Gan, G. Zhao, H. Xu et al., "Reverse vaccinology approach identify an Echinococcus granulosus tegumental membrane protein enolase as vaccine candidate," Parasitology Research, vol. 106, no. 4, pp. 873-882, 2010.

[51] V. Mundodi, A. S. Kucknoor, and J. F. Alderete, "Immunogenic and plasminogen-binding surface-associated $\alpha$-enolase of Trichomonas vaginalis," Infection and Immunity, vol. 76, no. 2, pp. 523-531, 2008. 
[52] C. Olver and M. Vidal, "Proteomic analysis of secreted exosomes," Subcellular Biochemistry, vol. 43, pp. 99-131, 2007.

[53] Y. Feng, X. Pan, W. Sun et al., "Streptococcus suis enolase functions as a protective antigen displayed on the bacterial cell surface," The Journal of Infectious Diseases, vol. 200, no. 10, pp. 1583-1592, 2009.

[54] J. Sha, T. E. Erova, R. A. Alyea et al., "Surface-expressed enolase contributes to the pathogenesis of clinical isolate SSU of Aeromonas hydrophila," Journal of Bacteriology, vol. 191, no. 9, pp. 3095-3107, 2009.

[55] A. Zhang, B. Chen, X. Mu et al., "Identification and characterization of a novel protective antigen, enolase of Streptococcus suis serotype 2," Vaccine, vol. 27, no. 9, pp. 1348-1353, 2009.

[56] D. F. Hoft and C. S. Eickhoff, "Type 1 immunity provides optimal protection against both mucosal and systemic Trypanosoma cruzi challenges," Infection and Immunity, vol. 70, no. 12, pp. 6715-6725, 2002.

[57] C. N. Paiva, M. T. L. Castelo-Branco, J. Lannes-Vieira, and C. R. Gattass, "Trypanosoma cruzi: protective response of vaccinated mice is mediated by $\mathrm{CD} 8+$ cells, prevents signs of polyclonal $\mathrm{T}$ lymphocyte activation, and allows restoration of a resting immune state after challenge," Experimental Parasitology, vol. 91, no. 1, pp. 7-19, 1999.

[58] R. T. Gazzinelli, I. P. Oswald, S. Hieny, S. L. James, and A. Sher, "The microbicidal activity of interferon- $\gamma$-treated macrophages againstTrypanosoma cruzi involves an L-arginine-dependent, nitrogen oxide-mediated mechanism inhibitable by interleukin-10 and transforming growth factor- $\beta$," European Journal of Immunology, vol. 22, no. 10, pp. 25012506, 1992.

[59] A. M. Abdelnoor, "Plasmid DNA vaccines," Current Drug Targets - Immune, Endocrine \& Metabolic Disorders, vol. 1, no. 1, pp. 79-92, 2001.

[60] G. Trinchieri, "Interleukin-10 production by effector T cells: Th1 cells show self control," Journal of Experimental Medicine, vol. 204, no. 2, pp. 239-243, 2007.

[61] J. L. Mege, S. Meghari, A. Honstettre, C. Capo, and D. Raoult, "The two faces of interleukin 10 in human infectious diseases," The Lancet Infectious Diseases, vol. 6, no. 9, pp. 557-569, 2006.

[62] Z. A. Andrade, S. G. Andrade, R. Correa, M. Sadigursky, and V. J. Ferrans, "Myocardial changes in acute Trypanosoma cruzi infection. Ultrastructural evidence of immune damage and the role of microangiopathy," American Journal of Pathology, vol. 144, no. 6, pp. 1403-1411, 1994.

[63] J. A. Marin-Neto, E. Cunha-Neto, B. C. Maciel, and M. V. Simoes, "Pathogenesis of chronic Chagas heart disease," Circulation, vol. 115, no. 9, pp. 1109-1123, 2007.

[64] H. Zapataestrella, C. Hummelnewell, G. Sanchezburgos et al., "Control of Trypanosoma cruzi infection and changes in Tcell populations induced by a therapeutic DNA vaccine in mice," Immunology Letters, vol. 103, no. 2, pp. 186-191, 2006.

[65] V. Tekiel, C. D. Alba-Soto, S. M. Gonzalez Cappa, M. Postan, and D. O. Sanchez, "Identification of novel vaccine candidates for Chagas' disease by immunization with sequential fractions of a trypomastigote cDNA expression library," Vaccine, vol. 27, no. 9, pp. 1323-1332, 2009.

[66] M. Arce-Fonseca, M. A. Ballinas-Verdugo, E. R. A. Zenteno et al., "Specific humoral and cellular immunity induced by Trypanosoma cruzi DNA immunization in a canine model," Veterinary Research, vol. 44, no. 1, p. 15, 2013.
[67] M. Esgleas, M. de la Cruz Dominguez-Punaro, Y. Li, J Ã.๑e. Harel, J. Daniel Dubreuil, and M. Gottschalk, "Immunization with SsEno fails to protect mice against challenge with Streptococcus suis serotype 2," FEMS Microbiology Letters, vol. 294, no. 1, pp. 82-88, 2009.

[68] A. O'Garra and P. Vieira, "TH1 cells control themselves by producing interleukin-10," Nature Reviews Immunology, vol. 7, no. 6, pp. 425-428, 2007. 


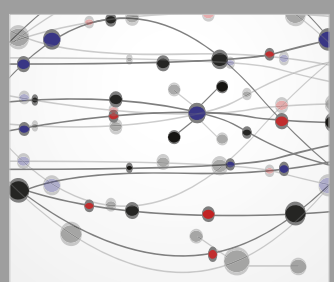

The Scientific World Journal
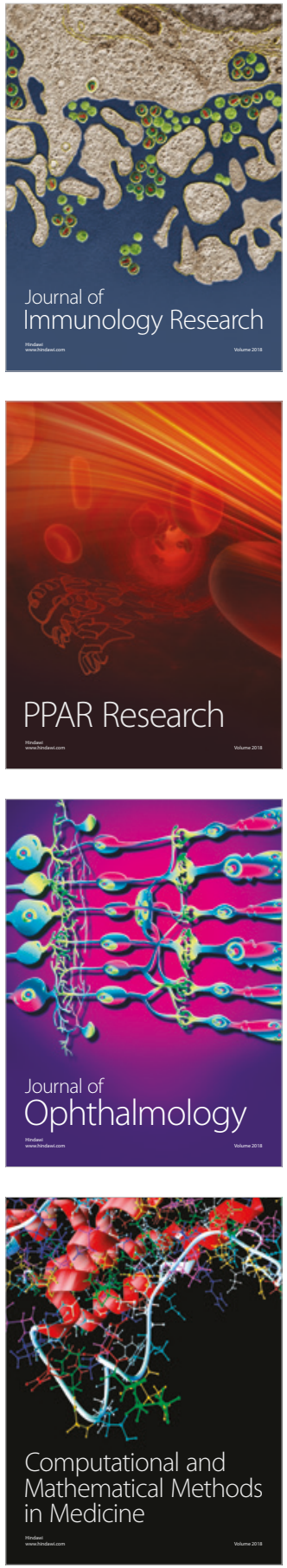

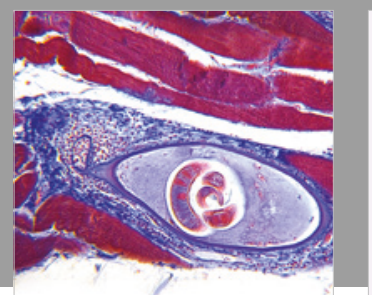

Gastroenterology Research and Practice

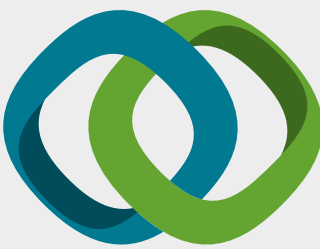

\section{Hindawi}

Submit your manuscripts at

www.hindawi.com
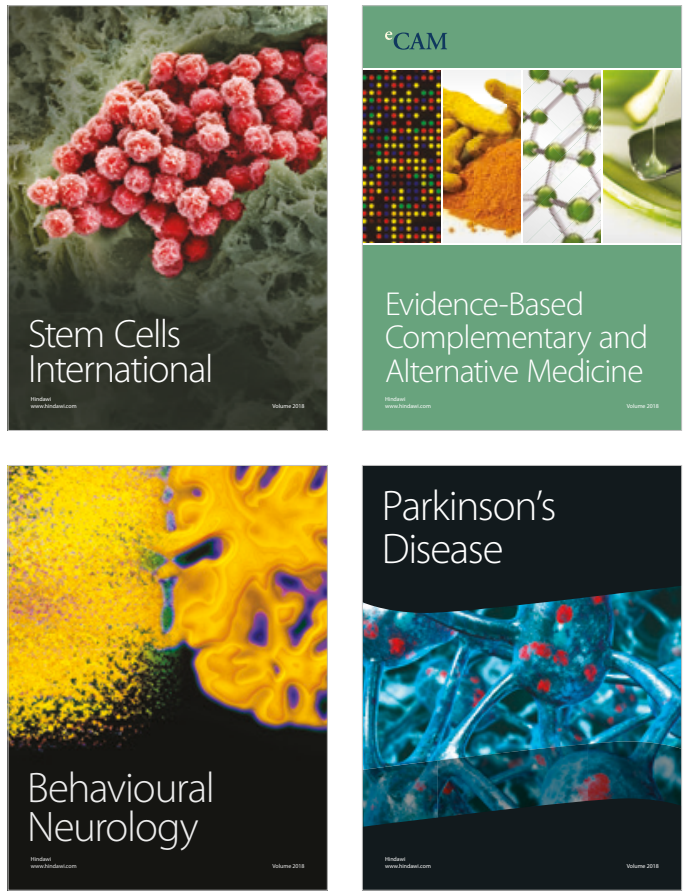

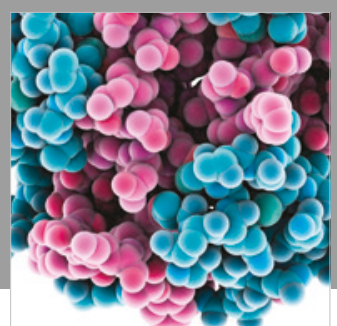

ournal of

Diabetes Research

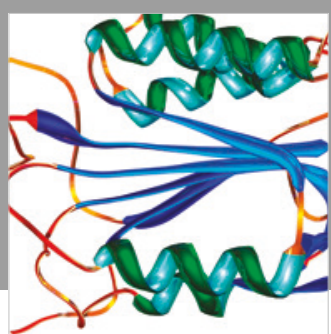

Disease Markers
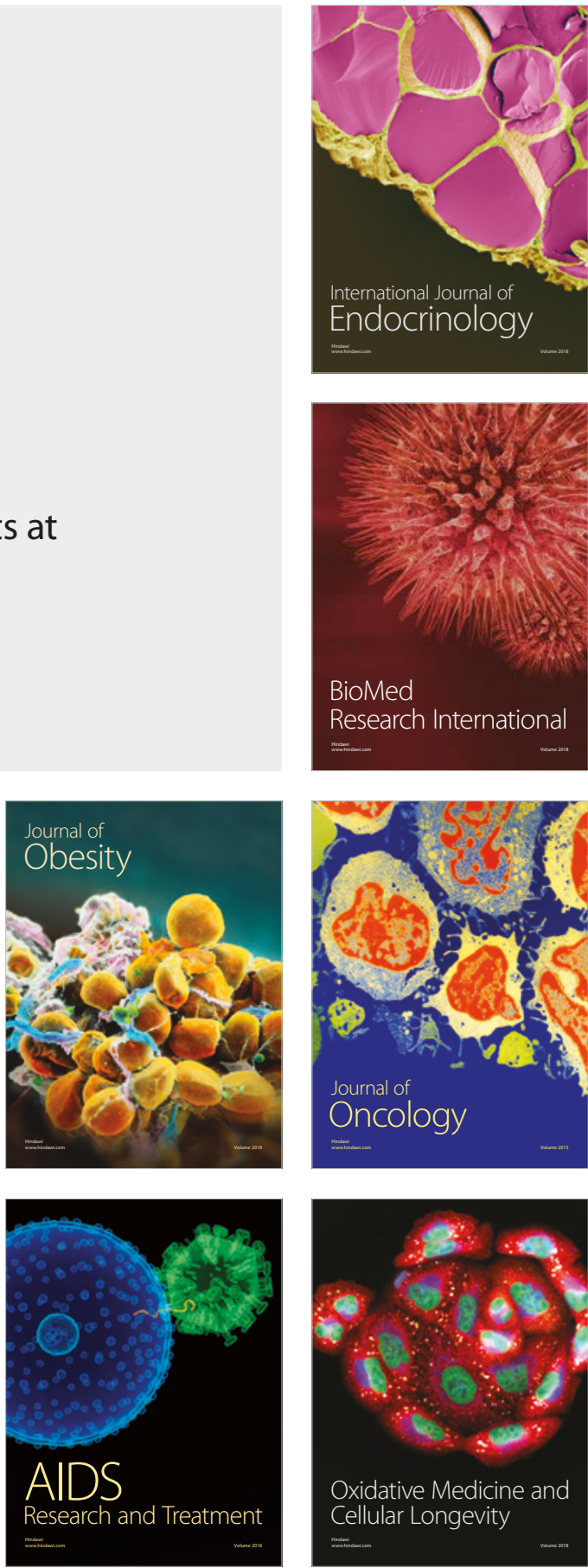